KUCP-0075

KUNS-1311 HE(TH)94/17

HEP-TH/9412093

December 1994

\title{
Complex-time path-integral formalism for quantum tunneling
}

\author{
HideAKi AOYAMA * \\ Department of Fundamental Sciences \\ Faculty of Integrated Human Studies \\ Kyoto University, Kyoto 606-01, Japan \\ and \\ TOSHIYUKi HARANO ${ }^{\dagger}$ \\ Department of Physics, Faculty of Science \\ Kyoto University, Kyoto 606-01, Japan
}

\begin{abstract}
The complex-time formalism is developed in the framework of the pathintegral formalism, to be used for analysis of the quantum tunneling phenomena. We show that subleading complex-time saddle-points do not account for the right WKB result. Instead, we develop a reduction formula, which enables us to construct Green functions from simple components of the potential, for which saddle-point method is applicable. This method leads us to the valid WKB result, which incorporates imaginary-time instantons and bounces, as well as the real-time boundary conditions.
\end{abstract}

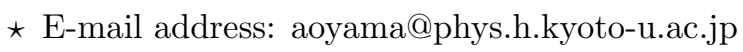

† E-mail address: harano@gauge.scphys.kyoto-u.ac.jp
} 


\section{Introduction}

The imaginary-time path-integral method has been successful for the treatment of the quantum tunneling phenomena in quantum field theories. The existence of the solution of the field equation, instantons and bounces, allows us to apply semiclassical approximation valid in small coupling.

In recent years, much effort has been made to develop this method further. One of the driving force was the hope of observing baryon and lepton number violation process in collision experiments in the $\mathrm{TeV}$ range. This process is associated with the tunneling between topologically different vacua in the standard electroweak model through the baryon and lepton number anomaly. 't Hooft ${ }^{[1]}$ first noticed this possibility, but at the same time found that the probability is highly suppressed. Later it was found that the potential barrier that separated the vacua has a "pass" (the Sphaleron), at the height of few TeV. ${ }^{[2]}$ This implies that even if the tunneling at the bottom of the potential well is highly suppressed as was estimated by 't Hooft, it may not be suppressed once initial energy is of order of $\mathrm{TeV}$. This expectation lead to series of works on developing the original instanton methods to be applicable to higher energy tunneling. This development has important implications in quantum theory in general, including nuclear fission problems as well as a number of tunneling problems in matter physics.

The original dilute-instanton-gas approximation is valid at the ground state. This can be confirmed by comparing the result in quantum mechanics with that of the WKB calculation. As the initial energy goes up, instanton result becomes invalid. In quantum mechanics, it fails in the estimate of the energy splitting of the first (and higher) excited state in double well. In quantum field theory, the cross sections violate the unitarity. ${ }^{[3]}$ One of the present authors (H.A.) and Kikuchi ${ }^{[4]}$ found that the interactions between the instantons become relevant: It gives the right energy splitting in quantum mechanics and satisfies the unitarity bound for the cross section.

As the energy yet goes up, the distances between instanton and anti-instanton 
decreases. Thus the semi-classical configurations are far from the solution of the equations of motion. Consequently, the valley methods ${ }^{[5]}$ were adopted. The new valley method ${ }^{[6]}$ was developed for this purpose in mind and yields configurations that allows exact conversion of the smallest eigenvalue to collective coordinate. Khoze and Ringwald ${ }^{[7]}$ used a series of approximate valley configurations to estimate the total tunneling cross section. The new valley method was recently applied to the bounce problem by one of the authors and Wada. They solved for the bounce-valley and identified small bubble configurations valid for the finite energy decay. ${ }^{[8]}$

All these analyses were carried out in the imaginary-time formalism. Thus basic as well as practical questions related to analytic continuation remain. How does the boundary condition matches that of the valley configurations? In estimating manypoint Green functions, how does the external fields enter (affect) the imaginary time configuration? [This is crucial for the validity of the path-deformations in the valley calculation, as well as bounce calculations.] These are some of the relevant questions. Some other points are elaborated by Boyanovsky, Willey and Holman. ${ }^{[9]}$ They also note that in some cases the imaginary-time method leads us to apparently contradictory results.

In quantum mechanics, the complex-time method has been studied by various authors. $^{[9-16]}$ It was argued that it allows semi-classical approximation for tunneling phenomena and overcomes various problems associated with the pure-imaginarytime method. ${ }^{[9]}$ In it, one considers the analytic continuation of the time-integral in Fourier transform of the Feynman kernel. The existence of the tunneling leads to the existence of the complex saddle-points in the time-plane. It was claimed that semi-classical approximation is done by deforming the time-integral so that it goes through all such saddle-points. ${ }^{[16]}$

This gives one hope that calculations in field theory may be improved by using the complex-time method. Indeed, Son and Rubakov ${ }^{[17-19]}$ adopted a complex-time method for the estimate of the cross section. They used periodic instanton-anti- 
instanton solution in imaginary time, and the initial Minkowski state overlaps with the instanton as a coherent state, much like the Aoyama and Goldberg ${ }^{[20]}$ calculation of the Sphaleron cross section.

Closer look, however, reveals many unanswered questions. ${ }^{[21]}$ It is known that the complex-time plane is plagued with singularities and infinite number of saddlepoints, which form lattice structure. Among saddle-points, there are two kinds, those that are solutions of the equation of motions (we shall call these "physical saddle-points" in this paper), and those that are not ("unphysical saddle-points"). How the path is deformed to avoid the singularities and to go through only the physical saddle-points is unknown. One simply assumes that all and only the physical saddle-points contribute. Even so, the weight of each saddle-point is a riddle. They are determined so that the result agrees with that of the WKB approximation. It is not known how it comes about from the path.

In this paper, we give the solution of these problems, based on a reduction formula in the number of the turning points of the path. In the next section, we give a brief overview of the saddle-point calculations in complex-time. There we elaborate some of the points briefly mentioned above. In section 3, we analyze the orthonormality of the WKB wavefunctions and construct the Green function from them. The connection formula for the WKB wavefunction is discussed in the section 4, where it leads to the reduction formula for Green function. Expanding this formula, we obtain a series, which can be interpreted as a sum over the physical saddle-points. The weights and the phases of saddle-points are determined from this formula. The last section gives summary and discussions. 


\section{Saddle-point method}

We consider one-dimensional quantum mechanics with action;

$$
S=\int d t\left[\frac{1}{2} \dot{x}^{2}-V(x)\right] .
$$

The potential $V(x)$ is assumed to be smooth enough to allow WKB approximation in asymptotic regions I $(x \rightarrow-\infty)$ and II $(x \rightarrow \infty)$.

The finite-time Green function (Feynman kernel) is defined by the following in the Heisenberg representation;

$$
G\left(x_{i}, x_{f} ; T\right)=\left\langle x_{f}\left|e^{-i H T}\right| x_{i}\right\rangle
$$

in terms of the hamiltonian of the system, $H$. The advanced and retarded resolvents are, respectively;

$$
\begin{aligned}
& G^{A}\left(x_{i}, x_{f} ; E\right)=i \int_{-\infty}^{0} d T e^{i(E-i \delta) T} G\left(x_{i}, x_{f} ; T\right)=\left\langle x_{f}\left|\frac{1}{E-i \delta-H}\right| x_{i}\right\rangle, \\
& G^{R}\left(x_{i}, x_{f} ; E\right)=-i \int_{0}^{\infty} d T e^{i(E+i \delta) T} G\left(x_{i}, x_{f} ; T\right)=\left\langle x_{f}\left|\frac{1}{E+i \delta-H}\right| x_{i}\right\rangle,
\end{aligned}
$$

where real-positive $\delta$ is introduced to guarantee the convergence of the integrals. The poles of these Green functions come from the bound states, and the cut from the continuous spectra.

Let us first look at the saddle-point approximation for the path integral,

$$
G\left(x_{i}, x_{f} ; T\right)=\int_{x(0)=x_{i}}^{x(T)=x_{f}} \mathcal{D} x e^{i S} .
$$


One obtains the following from the saddle-point method;

$$
G\left(x_{i}, x_{f} ; T\right)=\sum_{x_{c l}}\left[\frac{2 \pi}{i} \frac{\dot{x}_{c l}(0) \dot{x}_{c l}(T)}{-\partial^{2} S_{c l} / \partial T^{2}}\right]^{-1 / 2} e^{i S_{c l}}
$$

where $x_{c l}$ is the solution of the classical equation of motion and $S_{c l}$ is the action evaluated at the classical solution. This approximation is valid as long as both $x_{i}$ and $x_{f}$ are far from the turning points where classical velocities vanish. Substituting (2.5) into the retarded resolvent in (2.3), one arrives at the following expression;

$$
G^{R}\left(x_{i}, x_{f} ; E\right)=-i \int_{0}^{\infty} d T \sum_{x_{c l}}\left[\frac{2 \pi}{i} \frac{\dot{x}_{c l}(0) \dot{x}_{c l}(T)}{-\partial^{2} S_{c l} / \partial T^{2}}\right]^{-1 / 2} e^{i\left(E T+S_{c l}\right)} .
$$

In the above, we absorbed the infinitesimal imaginary convergence factor $(i \delta)$ in E.

In calculating (2.6), one hopes to apply the saddle-point approximation to the $T$-integral. The saddle-point condition (the stable-phase condition, to be more exact) is then,

$$
E=-\frac{\partial S_{c l}}{\partial T}=\frac{1}{2}{\dot{x_{c l}}}^{2}+V\left(x_{c l}\right)
$$

In other words, the solution of the equation of motion $x_{\mathrm{cl}}(t)$ has to have the energy E.

Let us consider a situation depicted in Fig.1. Both the initial point $x_{i}$ and the final point $x_{f}$ lies in the forbidden region. There is no real-time solution of the equation of motion. Thus no semi-classical approximation is possible for the real-time formalism.

If one assumes that the expression (2.5) gives correct analytic continuation of the Green function in the complex $T$-plane when complex- $T$ solutions of the 
equation of motion exist, one could go to the complex $T$-plane and apply the saddle-point method. One then arrives at the following expression;

$$
G\left(x_{i}, x_{f} ; E\right)=-i \sum_{T_{s}} \sum_{x_{c l}}\left|\dot{x}_{c l}(0) \dot{x}_{c l}\left(T_{s}\right)\right|^{-1 / 2} w\left(T_{s}\right) p\left(T_{s}\right) e^{i W_{c l}\left(T_{s}\right)}
$$

where $T_{s}$ is given by (2.7). Here $W_{c l}\left(T_{s}\right)$ is the WKB phase, $w\left(T_{s}\right)$ is weight and $p\left(T_{s}\right)$ is phase for the corresponding saddle-point. The original contour is deformed to pass the saddle-points by a series of steepest descent paths from the origin $T=0$ to the positive infinity on the real axes $(\operatorname{Re} T=\infty, \operatorname{Im} T=0)$. The weight of a saddle-point, $w\left(x_{c l}\right)$, is determined by how the contour crosses the saddle-point: If the contour does not cross the saddle-point, the corresponding weight is zero. If the contour crosses it along the steepest descent direction, the weight is 1 . In case the contour reaches a sub-dominant saddle-point from the direction orthogonal to the steepest descent and leaves it along the steepest descent direction, the weight is $1 / 2$ (as in the calculation of the false vacuum decay). The phase of a saddle-point, $p\left(T_{s}\right)$ comes from the square root in Eq.(2.6).

Carlitz and Nicole applied this method to linear potential, quadratic well and quadratic barrier, all of which are exactly solvable. Using the exact expression for the Green function (2.2), they found that the saddle-points explained above, namely the ones associated with the complex-time solution, indeed lead to saddlepoints in the $T$-plain [we shall call these "physical saddle-points"]. They, however found more: there were saddle-points that are not associated with any solutions of equations of motion (unphysical ones), and also singularities due to the periodicities. Deforming the $T$-integration path to avoid singularities, they found that the path indeed goes through only the physical saddle-points. As a result, the weights and phases in (2.8) are determined, which lead to the correct WKB result.

In the case of a double-well potential, the deformation of the integration contour is not specified, which is understandable in view of the fact that the analytic structure of (2.2) in complex $T$-plain is fairy complicated. The claim made in the 
literatures is simply that the contour passes all saddle-points that correspond to classical trajectories with weights that are extracted from the above calculation.

The problem lies in the fact that we do not know the contour which passes all physical saddle-points that are distributed in complex-time plane. Because of this, we cannot determine the weights of the saddle-points. In the case where $x_{i}$ and $x_{f}$ are in the forbidden region on the right side of the double-well potential as depicted in Fig.1, saddle-points in complex $T$-plane are given by

$$
\begin{array}{r}
T_{s}= \pm T\left(b_{2}, x_{i}\right) \pm T\left(b_{2}, x_{f}\right)+l T\left(a_{1}, b_{1}\right)+m T\left(b_{1}, a_{2}\right)+n T\left(a_{2}, b_{2}\right) \\
l, m, n=0, \pm 1, \pm 2, \ldots
\end{array}
$$

where

$$
T(x, y)=2 \int_{x}^{y} \frac{d x^{\prime}}{\sqrt{2\left(E-V\left(x^{\prime}\right)\right)}} .
$$

Notice that $T(x, y)$ is pure imaginary if $(x, y)$ is in a forbidden region. These saddlepoints $T_{s}$ are shown as solid circles in Fig.2. The unphysical saddle-points are also shown as open circles in the same figure. We know of no contour which passes only the physical ones and avoids the unphysical ones. Thus so far the complextime method has not been worked out for the double-well and more complicated potentials.

\section{Green function and the WKB wavefunctions}

We shall examine the Green functions by using the complete orthonormal set of the eigenfunctions $\left\{\left|\psi_{n}\right\rangle\right\}$ of $H$. For example, the retarded Green function in (2.3) is written as

$$
G^{R}\left(x_{i}, x_{f} ; T\right)=\sum_{n} \frac{\left\langle x_{f} \mid \psi_{n}\right\rangle\left\langle\psi_{n} \mid x_{i}\right\rangle}{E+i \delta-\lambda_{n}},
$$

where the sum over the complete set is actually made of the integration over the continuum spectrum and sum over the discrete bound states. 
In order to study of the Green functions in the asymptotic region, we use the WKB approximation for the wavefunctions $\psi(x)=\langle x \mid \psi\rangle$. For a state with the eigenvalue $\lambda$ in the continuum spectrum, the second-order WKB approximation yields the following;

$$
\psi_{\lambda}(x)=\frac{1}{\sqrt{2 \pi p(x)}} \times \begin{cases}\left(A_{\lambda} e^{i \int_{x}^{x_{1}} p\left(x^{\prime}\right) d x^{\prime}}+B_{\lambda} e^{-i \int_{x}^{x_{1}} p\left(x^{\prime}\right) d x^{\prime}}\right), & \text { for } x \in \mathrm{I}, \\ \left(C_{\lambda} e^{-i \int_{x_{2}}^{x} p\left(x^{\prime}\right) d x^{\prime}}+D_{\lambda} e^{i \int_{x_{2}}^{x} p\left(x^{\prime}\right) d x^{\prime}}\right), & \text { for } x \in \mathrm{II},\end{cases}
$$

where $p(x)=\sqrt{2(\lambda-V(x))}$. We choose the limits $x_{1}$ and $x_{2}$ of the integrations to be the nearest turning points for definiteness. Among the coefficients $A, B, C$, and $D$, only two are independent. Their inter-relation is linear due to the superposition principle and can be written as follows; ${ }^{[22]}$

$$
\left(\begin{array}{c}
A_{\lambda} \\
B_{\lambda}
\end{array}\right)=S(\lambda)\left(\begin{array}{c}
C_{\lambda} \\
D_{\lambda}
\end{array}\right)
$$

The $2 \times 2$ matrix $S(\lambda)$ is determined by the shape of the potential $V(x)$ in the intermediate region between $x_{1}$ and $x_{2}$. The flux conservation law is satisfied for the Schrödinger equation;

$$
\frac{d}{d x} j=0, \quad j=\frac{i}{2}\left(\frac{d \psi^{*}}{d x} \psi-\psi^{*} \frac{d \psi}{d x}\right) .
$$

Substituting the WKB expression (3.2) into the above, we find that

$$
j=\frac{1}{2 \pi}\left(|A|^{2}-|B|^{2}\right)=\frac{1}{2 \pi}\left(|C|^{2}-|D|^{2}\right)
$$

This results in the following relation between the matrix elements of $S(\lambda)$;

$$
\begin{gathered}
\left|S_{11}\right|^{2}-\left|S_{21}\right|^{2}=1, \quad\left|S_{12}\right|^{2}-\left|S_{22}\right|^{2}=1, \\
S_{11}^{*} S_{12}-S_{21}^{*} S_{22}=0 .
\end{gathered}
$$

As a result, the matrix $S(\lambda)$ is parametrized by three real functions $\alpha(\lambda), \beta(\lambda)$, 
and $\rho(\lambda)$ as follows;

$$
S(\lambda)=\left(\begin{array}{cc}
e^{i \alpha} \cosh \rho & e^{i \beta} \sinh \rho \\
e^{-i \beta} \sinh \rho & e^{-i \alpha} \cosh \rho
\end{array}\right)
$$

For a given eigenvalue $\lambda$, there are two independent eigenfunctions. In order to construct them, we look at the inner-product of the eigenfunction $\psi_{\lambda}(x)$ with coefficients $\left(A_{\lambda}, B_{\lambda}, C_{\lambda}, D_{\lambda}\right)$ and another eigenfunction $\tilde{\psi}_{\lambda^{\prime}}(x)$ with $\left(\tilde{A}_{\lambda^{\prime}}, \tilde{B}_{\lambda^{\prime}}, \tilde{C}_{\lambda^{\prime}}, \tilde{D}_{\lambda^{\prime}}\right)$. Integration in the asymptotic regions determines the coefficient of the delta function $\delta\left(\lambda-\lambda^{\prime}\right)$ completely, as the delta function can come only from the infinite integrations. [In order to calculate the finite terms we need to solve the Schrödinger equation completely. But this is not necessary for the current purpose.] In fact, integration in the region II yields,

$$
\begin{aligned}
& \int_{x_{2}}^{\infty} d x \psi_{\lambda}^{*}(x) \tilde{\psi}_{\lambda^{\prime}}(x)=\frac{1}{2 \pi} \int_{x_{2}}^{\infty} \frac{d x}{\sqrt{p(x) p^{\prime}(x)}} \\
& \quad \times\left(C_{\lambda}^{*} \tilde{C}_{\lambda^{\prime}} e^{i \int_{x_{2}}^{x}\left(p\left(x^{\prime}\right)-p^{\prime}\left(x^{\prime}\right)\right) d x^{\prime}}+D_{\lambda}^{*} \tilde{D}_{\lambda^{\prime}} e^{-i \int_{x_{2}}^{x}\left(p\left(x^{\prime}\right)-p^{\prime}\left(x^{\prime}\right)\right) d x^{\prime}}\right. \\
& \left.\quad+C_{\lambda}^{*} \tilde{D}_{\lambda^{\prime}} e^{i \int_{x_{2}}^{x}\left(p\left(x^{\prime}\right)+p^{\prime}\left(x^{\prime}\right)\right) d x^{\prime}}+D_{\lambda}^{*} \tilde{C}_{\lambda^{\prime}} e^{-i \int_{x_{2}}^{x}\left(p\left(x^{\prime}\right)+p^{\prime}\left(x^{\prime}\right)\right) d x^{\prime}}\right)
\end{aligned}
$$

As we are interested in the singularity of the above for $\lambda=\lambda^{\prime}$, we expand the exponent using the following for $\lambda \sim \lambda^{\prime}$,

$$
p\left(x^{\prime}\right)-p^{\prime}\left(x^{\prime}\right) \simeq \frac{\left(\lambda-\lambda^{\prime}\right)}{p\left(x^{\prime}\right)}
$$

By using a new coordinate $y$ defined by $d y=d x / p(x)$, we find the contribution 
from the first two terms to the delta function;

$$
\begin{aligned}
\int_{x_{2}}^{\infty} d x \psi_{\lambda}^{*}(x) \tilde{\psi}_{\lambda^{\prime}}(x) & =\frac{1}{2 \pi} \int_{y_{2}}^{\infty} d y\left(C_{\lambda}^{*} \tilde{C}_{\lambda} e^{i\left(\lambda-\lambda^{\prime}\right)\left(y-y_{2}\right)}+D_{\lambda}^{*} \tilde{D}_{\lambda} e^{-i\left(\lambda-\lambda^{\prime}\right)\left(y-y_{2}\right)}\right)+\ldots \\
& =\frac{1}{2}\left(C_{\lambda}^{*} \tilde{C}_{\lambda}+D_{\lambda}^{*} \tilde{D}_{\lambda}\right) \delta\left(\lambda-\lambda^{\prime}\right)+\ldots
\end{aligned}
$$

where we have neglected all the finite terms. Doing the similar calculation for the region $\mathrm{I}$, we find

$$
\int_{-\infty}^{\infty} d x \psi_{\lambda}^{*}(x) \tilde{\psi}_{\lambda^{\prime}}(x)=\frac{1}{2}\left(A_{\lambda}^{*} \tilde{A}_{\lambda}+B_{\lambda}^{*} \tilde{B}_{\lambda}+C_{\lambda}^{*} \tilde{C}_{\lambda}+D_{\lambda}^{*} \tilde{D}_{\lambda}\right) \delta\left(\lambda-\lambda^{\prime}\right) .
$$

Using the above result, we choose our orthonormal eigenfunctions for a given $\lambda$ as the following;

$$
\begin{aligned}
& \left(A_{\lambda}, B_{\lambda}, C_{\lambda}, D_{\lambda}\right)^{(1)}=\left(\frac{e^{i \alpha}}{\cosh \rho}, 0,1,-e^{i(\alpha-\beta)} \tanh \rho\right), \\
& \left(A_{\lambda}, B_{\lambda}, C_{\lambda}, D_{\lambda}\right)^{(2)}=\left(e^{i(\alpha+\beta)} \tanh \rho, 1,0, \frac{e^{i \alpha}}{\cosh \rho}\right) .
\end{aligned}
$$

[The above corresponds to two incoming states. There are of course other choices, such as stationary states, but all of those reads to the same result for the Feynman kernel.]

Let us first look at the case when both $x_{i}$ and $x_{f}$ are in an allowed region in II. From (3.12), we find that

$$
\begin{aligned}
\sum_{i=1,2} \psi_{\lambda}^{(i)}\left(x_{f}\right) & \psi_{\lambda}^{(i) *}\left(x_{i}\right)=\frac{1}{2 \pi \sqrt{p\left(x_{i}\right) p\left(x_{f}\right)}} \\
\times & {\left.\left[e^{i \int_{x_{i}}^{x_{f}} p\left(x^{\prime}\right) d x^{\prime}}-e^{i\left(\int_{x_{2}}^{x_{i}} p\left(x^{\prime}\right) d x^{\prime}+\int_{x_{2}}^{x_{f}} p\left(x^{\prime}\right) d x^{\prime}\right)} e^{i(\alpha-\beta)} \tanh \rho+\text { (c.c. }\right)\right] }
\end{aligned}
$$

The complex conjugate part can be understood as the same function below the cut 
on the real-axis of the complex $\lambda$-plane (see Fig.3). This is guaranteed by the fact that as $\lambda$ moves below the cut, the role of the coefficients $A$ and $B$ is exchanged and likewise for $C$ and $D$, and as a result, the phases $\alpha$ and $\beta$ change their signs, while $\rho$ does not. Therefore the sum over the continuous spectra in the Feynman kernel (3.1) is written as the following $\lambda$-integration;

$$
\begin{aligned}
& G^{R}\left(x_{i}, x_{f} ; E\right)=\frac{1}{2 \pi} \int_{C} d \lambda \frac{1}{E+i \delta-\lambda} \frac{1}{\sqrt{p\left(x_{i}\right) p\left(x_{f}\right)}} \\
& \quad \times \begin{cases}e^{i \int_{x_{2}}^{x_{i}} p\left(x^{\prime}\right) d x^{\prime}}\left[e^{-i \int_{x_{2}}^{x_{f}} p\left(x^{\prime}\right) d x^{\prime}}-e^{i \int_{x_{2}}^{x_{f}} p\left(x^{\prime}\right) d x^{\prime}} e^{i(\alpha-\beta)} \tanh \rho\right] & \text { for } x_{i}>x_{f} \\
e^{i \int_{x_{2}}^{x_{f}} p\left(x^{\prime}\right) d x^{\prime}}\left[e^{-i \int_{x_{2}}^{x_{i}} p\left(x^{\prime}\right) d x^{\prime}}-e^{i \int_{x_{2}}^{x_{i}} p\left(x^{\prime}\right) d x^{\prime}} e^{i(\alpha-\beta)} \tanh \rho\right] & \text { for } x_{f}>x_{i} .\end{cases}
\end{aligned}
$$

The integration contour $C$ in the complex $\lambda$-plane is as in Fig.3. In (3.14), we have chosen the integrand so that it converges for $|\lambda| \rightarrow \infty$, which we need later. As for the discreet spectrum, it is determined by the absence of the diverging behaviour of the wavefunction, which translates into the condition that for $C=0, A=0$. From (3.7), we thus find that $\sinh \rho=0$ when $\lambda$ is equal to a discreet eigenvalue of $H$. This allows us to write the sum over the discreet spectra as pole integrations of the second term in (3.14) depicted in Fig.3. Connecting all the contours and closing it at the infinity, we find that the whole contour enclose the pole at $\lambda=E+i \delta$. Thus we end up with the expression

$$
\begin{aligned}
G^{R}\left(x_{i}, x_{f} ; E\right) & =-\frac{i}{\sqrt{p\left(x_{i}\right) p\left(x_{f}\right)}} \\
& \times \begin{cases}e^{i \int_{x_{2}}^{x_{i}} p\left(x^{\prime}\right) d x^{\prime}}\left(e^{-i \int_{x_{2}}^{x_{f}} p\left(x^{\prime}\right) d x^{\prime}}+i R e^{i \int_{x_{2}}^{x_{f}} p\left(x^{\prime}\right) d x^{\prime}}\right) & \text { for } x_{i}>x_{f}, \\
e^{i \int_{x_{2}}^{x_{i}} p\left(x^{\prime}\right) d x^{\prime}}\left(e^{-i \int_{x_{2}}^{x_{f}} p\left(x^{\prime}\right) d x^{\prime}}+i R e^{i \int_{x_{2}}^{x_{f}} p\left(x^{\prime}\right) d x^{\prime}}\right) & \text { for } x_{f}>x_{i} .\end{cases}
\end{aligned}
$$

where we defined the "reflection coefficient" $R$ by the following,

$$
R=i \frac{S_{21}}{S_{22}}=i e^{i(\alpha-\beta)} \tanh \rho .
$$


We note that this expression (3.15) is valid for a general value of $E$ with proper analytic continuation of the coefficients. Therefore, if any end-points are in a forbidden region, simple analytic continuation of (3.15) is appropriate. We shall use this result in the next section.

\section{Reduction formula and its expansion}

In this section, we derive a formula for evaluating the Green function in a system with an arbitrary potential to which we can apply the WKB approximation. We consider a general potential which has arbitrary number of wells, and show that the Green function is given by summing up all contributions of classical paths.

Let us consider the case where $x_{i}$ and $x_{f}$ lie in the forbidden region on the same sides of the wells. The potential which has $n$ wells is depicted in Fig.4. By the analytical continuation of (3.15) in $E$, we find the retarded resolvent in the following form;

$$
G^{R}\left(x_{i}, x_{f} ; E\right)=-\left|p\left(x_{i}\right) p\left(x_{f}\right)\right|^{-1 / 2} e^{-\Delta_{i}}\left(e^{\Delta_{f}}+i R_{n} e^{-\Delta_{f}}\right)
$$

where

$$
\Delta_{i, f} \equiv \int_{b_{n}}^{x_{i, f}} d x|p(x)|
$$

In order to specify the fact that this expression is for $n$ wells, we attach the subscript $(n)$ to the coefficients hereafter.

Due to the existence of the intermediate region $\left(b_{n-1}, a_{n}\right)$, the matrix $S^{(n)}$, which connects the regions I and II, can be written in terms of the matrices $S^{(n-1)}$ that connects regions I and $\left(b_{n-1}, a_{n}\right)$ and $\tilde{S}$ for $\left(b_{n-1}, a_{n}\right)$ and II. If we apply the linear WKB connection formula for the latter region, which we can obtain from the saddle-point method for a linear potential, we obtain the following;

$$
S^{(n)}(E)=S^{(n-1)}(E)\left(\begin{array}{cc}
e^{-\Delta_{n-1}} & 0 \\
0 & e^{\Delta_{n-1}}
\end{array}\right)\left(\begin{array}{cc}
\frac{i}{2} & -\frac{i}{2} \\
1 & 1
\end{array}\right)
$$




$$
\begin{array}{r}
\times\left(\begin{array}{cc}
e^{-i\left(W_{n}+\frac{\pi}{2}\right)} & 0 \\
0 & e^{i\left(W_{n}+\frac{\pi}{2}\right)}
\end{array}\right)\left(\begin{array}{cc}
\frac{1}{2} & i \\
\frac{1}{2} & -i
\end{array}\right) \\
=S^{(n-1)}(E)\left(\begin{array}{cc}
\frac{1}{2} e^{-\Delta_{n-1}} \cos W_{n} & e^{-\Delta_{n-1}} \sin W_{n} \\
-e^{\Delta_{n-1}} \sin W_{n} & 2 e^{\Delta_{n-1}} \cos W_{n}
\end{array}\right)
\end{array}
$$

where $W_{n}$ and $\Delta_{n-1}$ are defined by,

$$
\begin{gathered}
W_{n}=\int_{a_{n}}^{b_{n}} d x|p(x)|, \\
\Delta_{n-1}=\int_{b_{n-1}}^{a_{n}} d x|p(x)| .
\end{gathered}
$$

Therefore, we find the relations;

$$
\begin{gathered}
S_{21}^{(n)}=\frac{1}{2} S_{21}^{(n-1)} e^{-\Delta_{n-1}} \cos W_{n}-S_{22}^{(n-1)} e^{\Delta_{n-1}} \sin W_{n} \\
S_{22}^{(n)}=S_{21}^{(n-1)} e^{-\Delta_{n-1}} \sin W_{n}+2 S_{22}^{(n-1)} e^{\Delta_{n-1}} \cos W_{n} .
\end{gathered}
$$

From (3.16), (4.6) and (4.7), we find that $R_{n}$ can be written as the following;

$$
R_{n}=\frac{i}{2} \frac{-\sin W_{n}-\frac{i}{2} e^{-2 \Delta_{n-1}} R_{n-1} \cos W_{n}}{\cos W_{n}-\frac{i}{2} e^{-2 \Delta_{n-1}} R_{n-1} \sin W_{n}}
$$

We find it most convenient to rewrite this to the following two expressions;

$$
\begin{gathered}
R_{n}=\frac{1}{2} \frac{1-\tilde{R}_{n} e^{2 i W_{n}}}{1+\tilde{R}_{n} e^{2 i W_{n}}}, \\
\tilde{R}_{n}=\frac{1-\frac{1}{2} R_{n-1} e^{-2 \Delta_{n-1}}}{1+\frac{1}{2} R_{n-1} e^{-2 \Delta_{n-1}}} .
\end{gathered}
$$

Defined in this manner, the function $R_{n-1}$ corresponds to the reflection amplitude at the turning point $b_{n-1}$. 
In order to see the correspondence between these expressions and the saddlepoint method, let us expand (4.9) in an infinite series as follows;

$$
i R_{n}=\frac{i}{2}+\left(-i \tilde{R}_{n}\right) e^{2 i W_{n}}+\left(-i \tilde{R}_{n}\right)^{2}(-i) e^{4 i W_{n}}+\ldots
$$

The convergence of this series is guaranteed by the implicit factor $i \delta$. The retarded resolvent is then,

$$
\begin{aligned}
& G^{R}\left(x_{i}, x_{f} ; E\right)=-\left|p\left(x_{i}\right) p\left(x_{f}\right)\right|^{-1 / 2} \\
& \times\left[e^{-\left(\Delta_{i}-\Delta_{f}\right)}+\left\{\frac{i}{2}+\left(-i \tilde{R}_{n}\right) e^{2 i W_{n}}+\left(-i \tilde{R}_{n}\right)^{2}(-i) e^{4 i W_{n}}+\ldots\right\} e^{-\left(\Delta_{i}+\Delta_{f}\right)}\right] .
\end{aligned}
$$

This is the expression to be compared to the saddle-point expression (2.6). If we choose $T$ to be negative imaginary, $-i \tau$, the factor in the exponent is,

$$
i\left(E T+S_{c l}\right)=E \tau-S_{E}=-\int d \tau\left(\frac{d x}{d \tau}\right)^{2}=-\int d x|p(x)|
$$

Therefore, the two factors $\Delta_{i, f}$ is equal to the above quantity for the path from $x_{i, f}$ to the turning point $b_{n}$. The first term in (4.12) corresponds the contribution of the pure-imaginary-time path that starts from $x_{i}$ and reaches $x_{f}$ directly. The factor $e^{-\left(\Delta_{i}+\Delta_{f}\right)}$ in the rest of the terms is for the path from $x_{i}$ to the turning point $b_{n}$ and then from $b_{n}$ to $x_{f}$. The expansion of $i R_{n}$ is understood as contributions of the paths that oscillate in the allowed region $\left(a_{n}, b_{n}\right)$. Various factors have unique interpretations as factors coming from the turning points. This is most conveniently depicted in Fig.5. Similarly to (4.11), the expression (4.10) is expanded as the following,

$$
-i \tilde{R}_{n}=-i+\left(i R_{n-1}\right) e^{-2 \Delta_{n-1}}+\left(i R_{n-1}\right)^{2}\left(\frac{i}{2}\right) e^{-4 \Delta_{n-1}}+\ldots
$$

The corresponding diagrams are illustrated in Fig.6. 
We have so far derived the expressions that results from the WKB approximation for the $n$-th well. This procedure can be applied recursively for the rest of the wells. At the end, we are left with $R_{1}$, which is given by

$$
i R_{1}=\frac{1}{2} \tan W_{1}=\frac{i}{2}+(-i) e^{-2 i W_{1}}+(-i)^{3} e^{-4 i W_{1}}+\ldots
$$

where $W_{1}$ is defined by (4.4) by replacing $n$ with 1 . Therefore we find that $R_{1}$ is expressed as a sum of the contribution of the classical paths which evolve in the first well.

Combining (4.12), (4.14), and (4.15), we find all complex-time paths are included in the expression of the resolvent $G^{R}$. Therefore, the resulting Green function in a $n$-well potential is given by

$$
G^{R}\left(x_{i}, x_{f} ; E\right)=-\left|p\left(x_{i}\right) p\left(x_{f}\right)\right|^{-1 / 2} \sum_{x_{c l}} f\left(x_{c l}\right) e^{i W\left(x_{i}, x_{f} ; E\right)} .
$$

where $f\left(x_{c l}\right)$ is determined by the number of reflections and transmissions which the classical path contains, and $W\left(x_{i}, x_{f} ; E\right)$ is determined by what wells the path crosses and what barriers it tunnels through. The rules for calculating $f\left(x_{c l}\right)$ is the following: When a path has a reflection in the allowed region, it obtains $-i$. In the case of a reflection in the forbidden region, it gets $\frac{i}{2}$.

Let us next examine the case when $x_{i}, x_{f}$ are on opposite sides of the wells. Applying the analysis similar to the one in the section 3, we find that the Green function is expressed as follows;

$$
G^{R}\left(x_{i}, x_{f} ; E\right)=-\left|p\left(x_{i}\right) p\left(x_{f}\right)\right|^{-1 / 2} T_{n} e^{-\Delta_{i}-\Delta_{f}}
$$

where $T_{n}$ is the (analytically-continued) transmission amplitude

$$
T_{n}=\frac{1}{S_{22}^{(n)}} .
$$

Just as the previous case, we can express $T_{n}$ in terms of $T_{n-1}$ as in the following;

$$
T_{n}=\frac{1}{S_{21}^{(n-1)} e^{-\Delta_{n-1}} \sin W_{n}+2 S_{22}^{(n-1)} e^{\Delta_{n-1}} \cos W_{n}}
$$




$$
\begin{aligned}
& =\frac{T_{n-1} e^{-\Delta_{n-1}+i W_{n}}}{1+\frac{1}{2} R_{n-1} e^{-2 \Delta_{n-1}}+e^{2 i W_{n}}\left(1-\frac{1}{2} R_{n-1} e^{-2 \Delta_{n-1}}\right)} \\
& =\frac{e^{-\Delta_{n-1}}}{1+\frac{1}{2} R_{n-1} e^{-2 \Delta_{n-1}}} \frac{e^{i W_{n}}}{1+\tilde{R}_{n} e^{2 i W_{n}}} T_{n-1} .
\end{aligned}
$$

In the above, we have used (3.16), (4.7) and (4.10). Let us show that $T_{n}$ consists of all contributions of classical paths. The second factor in (4.19) is expanded in the following way,

$$
\frac{e^{i W_{n}}}{1+\tilde{R}_{n} e^{2 i W_{n}}}=e^{i W_{n}}+(-i)\left(-i \tilde{R}_{n}\right) e^{3 i W_{n}}+(-i)^{2}\left(-i \tilde{R}_{n}\right)^{2} e^{5 i W_{n}}+\ldots
$$

which corresponds to the diagrams in Fig.7 (a). The first factor in (4.19) is expanded as,

$\frac{e^{-\Delta_{n-1}}}{1+\frac{1}{2} R_{n-1} e^{-2 \Delta_{n-1}}}=e^{-\Delta_{n-1}}+\left(\frac{i}{2}\right)\left(i R_{n-1}\right) e^{-3 \Delta_{n-1}}+\left(\frac{i}{2}\right)^{2}\left(i R_{n-1}\right)^{2} e^{-5 \Delta_{n-1}}+\ldots$

which is illustrated in Fig.7 (b). As the previous case, repeating this procedure, we reach $T_{1}$, which is given by

$$
\begin{aligned}
T_{1} & =\frac{1}{2 \cos W_{1}} \\
& =e^{i W_{1}}+(-i)^{2} e^{3 i W_{1}}+(-i)^{4} e^{5 i W_{1}}+\ldots
\end{aligned}
$$

Therefore we conclude that $T_{n}$ consists of all contributions of classical paths.

The above analysis can be applied to other cases with various locations of $x_{i}$ and $x_{f}$. Thus we verify that thus constructed resolvent can be always interpreted as sum over the complex-time classical paths.

This formalism is valid for a metastable system and scattering process (Fig.8) as well as for a stationary system. It is apparent from this derivation that this complex-time method reproduces the result of the WKB approximation with the 
linear connection formula. The potential we consider is given in Fig.8(a). The turning points are labeled in the figure by $a_{1}, b_{1}$, and $a_{2}$. First we consider the case when $x_{i}$ is in the well and $x_{f}$ is outside of the barrier. The analysis similar to the above yields the Green function as in the following;

$$
G^{R}\left(x_{i}, x_{f} ; E\right)=-i\left|p\left(x_{i}\right) p\left(x_{f}\right)\right|^{-1 / 2} \frac{\left(e^{-i W_{i}}-i e^{i W_{i}}\right) e^{i W_{1}-\Delta_{1}+i W_{f}}}{\left(1+\tilde{R} e^{2 i W_{1}}\right)\left(1+\frac{1}{4} e^{-2 \Delta_{1}}\right)}
$$

where $\tilde{R}$ is given by

$$
\begin{aligned}
-i \tilde{R} & =-i \frac{1-\frac{1}{4} e^{-2 \Delta_{1}}}{1+\frac{1}{4} e^{-2 \Delta_{1}}} \\
& =-i+\frac{i}{2} e^{-2 \Delta_{1}}+\left(\frac{i}{2}\right)^{3} e^{-4 \Delta_{1}}+\ldots
\end{aligned}
$$

From (4.23) and (4.24), we find again that the resolvent is equal to the sum over the physical saddle-points. We find that the poles of the Green function are determined by the following;

$$
1+e^{2 i W_{1}}+\frac{1}{4} e^{-2 \Delta_{1}}\left(1-e^{2 i W_{1}}\right)=0
$$

Let us solve this equation iteratively. Then we obtain

$$
W_{1}(E)=\left(n+\frac{1}{2}\right) \pi-\frac{i}{4} e^{-2 \Delta_{1}}
$$

From (4.26), we find an imaginary part of the energy eigenvalue

$$
\operatorname{Im} E_{n}=-\frac{i}{2 T\left(E_{n}\right)} e^{-2 \Delta_{1}}
$$

where $T\left(E_{n}\right)$ is the period of the classical path between the turning points $a_{1}, b_{1}$

$$
T(E)=2 \int_{a_{1}}^{b_{1}} \frac{d x}{\sqrt{2(E-V(x))}}
$$

We find the decay rate of this metastable system. We note that the factor $1 / 2$ in (4.27) comes from the weight $1 / 2^{n}$ of the saddle-points with $n$ reflections in the forbidden region. 
Let us apply this method to a scattering process against a potential barrier in Fig.8. When $x_{i}$ and $x_{f}$ are separated by the barrier, the Green function is given by

$$
\begin{aligned}
G^{R}\left(x_{i}, x_{f} ; E\right) & =-i\left|p\left(x_{i}\right) p\left(x_{f}\right)\right|^{-1 / 2} \tilde{T} e^{i\left(W_{i}+W_{f}\right)} \\
& =-i\left|p\left(x_{i}\right) p\left(x_{f}\right)\right|^{-1 / 2} \sum_{k=1}^{\infty} e^{i\left(W_{i}+W_{f}\right)-(2 k-1) \Delta_{1}}\left(\frac{i}{2}\right)^{2(k-1)},
\end{aligned}
$$

where we have used the WKB expression for the transmission coefficient $\tilde{T}$;

$$
\tilde{T}=\frac{e^{-\Delta_{1}}}{1+\frac{1}{4} e^{-2 \Delta_{1}}}
$$

When $x_{i}$ and $x_{f}$ are on the same side, the Green function is given by

$$
\begin{aligned}
& G^{R}\left(x_{i}, x_{f} ; E\right)=-i\left|p\left(x_{i}\right) p\left(x_{f}\right)\right|^{-1 / 2} e^{i W_{i}}\left[e^{-i W_{f}}-i \tilde{R} e^{i W_{f}}\right] \\
& =-i\left|p\left(x_{i}\right) p\left(x_{f}\right)\right|^{-1 / 2} e^{i W_{i}}\left[e^{-i W_{f}}+\frac{i}{2} e^{i W_{f}}+\sum_{k=1}^{\infty} e^{i W_{f}-2 k \Delta_{1}}(-i)^{2 k-1}\right],
\end{aligned}
$$

where we have used the expression for the reflection amplitude,

$$
-i \tilde{R}=-i \frac{1-\frac{1}{4} e^{-2 \Delta_{1}}}{1+\frac{1}{4} e^{-2 \Delta_{1}}} .
$$

The coefficients $\tilde{T}$ and $\tilde{R}$ satisfy the unitarity condition

$$
|\tilde{T}|^{2}+|\tilde{R}|^{2}=1
$$

We again confirm the validity of the sum over the physical saddle-points. The weight $1 / 2^{n}$ is crucial for (4.33). 


\section{Discussion}

In the paper, we have given the re-formulation of the complex-time method. Using the connection formula for the wavefunctions, we constructed the reduction formula in the number of the turning points for the Green function. This yields series expansions, which can be understood as sum over in the classical complextime trajectory. This is understood as a sum over the physical saddle-points with specific weights and phases in the path-integral method. This shows the validity of the method proposed before, in the context of the path-integral method. We confirmed that this method yields results identical with that of the WKB approximation. Thus our construction gives solid basis for the starting point of the complex-time method.

What happens if one tries to extend this method to field theories? Not much is known. Rubakov et.al. basically assumes that the behavior of the Green functions in asymptotic region $(T \rightarrow \infty)$ does not depend on the imaginary part of $T$ and calculate with non-zero $\operatorname{Im} T$ so that the saddle-point approximation can be applied. However, even for Re $T \rightarrow \infty$, the Green function does depend on the $\operatorname{Im} T$, as we are allowed to have more configurations along the imaginary-time axis as $\operatorname{Im} T$ increases. Thus rather careful consideration would be required. The complex-time method instead relies on the $T$ integration as we have seen in this method. Thus somewhat different analysis may be required. One of them could be the combination of the valley method and the current complex-time method. The valley method can used to identify the imaginary-time tunneling paths, which are converted to collective coordinates. Thus, while the incoming particle are expressed in real-time expressions, the tunneling part may be obtained as the imaginary part of the complex-time development along the valley trajectory. There are rather interesting possibilities along this line, which should be further investigated. 


\section{REFERENCES}

1. G. 't Hooft, Phys. Rev. D14 (1976), 3432.

2. N. S. Manton, Phys. Rev. D28 (1983), 2019; F. R. Klinkhamer and N. S. Manton, Phys. Rev. D30 (1984), 2212.

3. A. Ringwald, Nucl. Phys. B330 (1989), 1; O. Espinosa, Nucl. Phys. B343 (1990), 310 .

4. H. Aoyama and H. Kikuchi, Phys. Lett. B247 (1990), 75, Phys. Rev. D43 (1991), 1999, Int. J. Mod. Phys. A7 (1992), 2741.

5. A. V.Yung, Nucl. Phys. B191 (1981), 47.

6. H. Aoyama and H. Kikuchi, Nucl. Phys. B369 (1992), 219.

7. V.V. Khoze and A. Ringwald, Nucl. Phys. B355 (1991), 351.

8. H. Aoyama and S. Wada, Kyoto University preprint KUCP-0069 (1994).

9. D. Boyanovsky, R. Willey and R. Holman, Nucl. Phys. B376 (1992), 599.

10. D. W. McLaughlin, J. Math. Phys. 13 (1972), 1099.

11. I. Bender, D. Gomez, H. Rothe and K. Rothe, Nucl. Phys. B136 (1978), 259.

12. S. Levit, J. W. Negele and Z. Paltiel, Phys. Rev. C22 (1980), 1979.

13. A. Patrascioiu, Phys. Rev. D24 (1981), 496.

14. A. Lapedes and E. Mottola, Nucl. Phys. B203 (1982), 58.

15. U. Weiss and W. Haeffner, Phys. Rev. D27 (1983), 2916.

16. R. D. Carlitz and D. A. Nicole, Ann. Phys. 164 (1985), 411.

17. S. Y. Khlebnikov, V. A. Rubakov and P. G. Tinyakov, Nucl. Phys. B367 (1991), 334 .

18. V. A. Rubakov, D. T. Son and P. G. Tinyakov, Phys. Lett. 287 (1992), 342. 
19. D. T. Son and V. A. Rubakov, Nucl. Phys. B424 (1994), 55.

20. H. Aoyama and H. Goldberg, Phys. Rev. Lett. 188B (1987), 506.

21. H. Aoyama and T. Harano, Kyoto University preprint, KUCP-0072/KUNS$1302(1994)$

22. H. Aoyama and M. Kobayashi, Prog. Theor. Phys. 64 (1980), 1045. 


\section{FIGURE CAPTIONS}

1) A potential $V(X)$ with asymptotic regions I and II.

2) Positions of saddle-points in complex $T$-plane.

3) The complex-plane of the eigenvalue $\lambda$. The cut on the real-axis is the continuous spectra and the poles are the bound states. The integration contour $C$ is for the continuous part of the Green function. The discreet part can be represented as integration around poles.

4) A potential with $n$ wells we consider. The turning points are denoted by $a_{i}$ and $b_{i}$, for left and right of the $i$-th well, respectively $(i=1 \sim n)$.

5) Diagrammatic representation of the expansion of $i R_{n}$. The first term corresponds to the direct reflection at the turning point $b_{n}$. Each path in the rest corresponds to the group of paths that have a number of oscillation in the $n$-th well. The information of the region left of $a_{n}$ is contained in $-i \tilde{R}_{n}$.

6) Diagrammatic representation of the expansion of $-i \tilde{R}_{n}$. The each path corresponds to a group of paths with different number of oscillation in the forbidden region $\left(b_{n-1}, a_{n}\right)$.

7) Classical paths traversing in the $n$-th well and tunneling through the $(n-1)$ th barrier. These are again expanded into groups of paths with different oscillations in either the allowed or forbidden regions.

8) A metastable system and a scattering process against a potential barrier. 


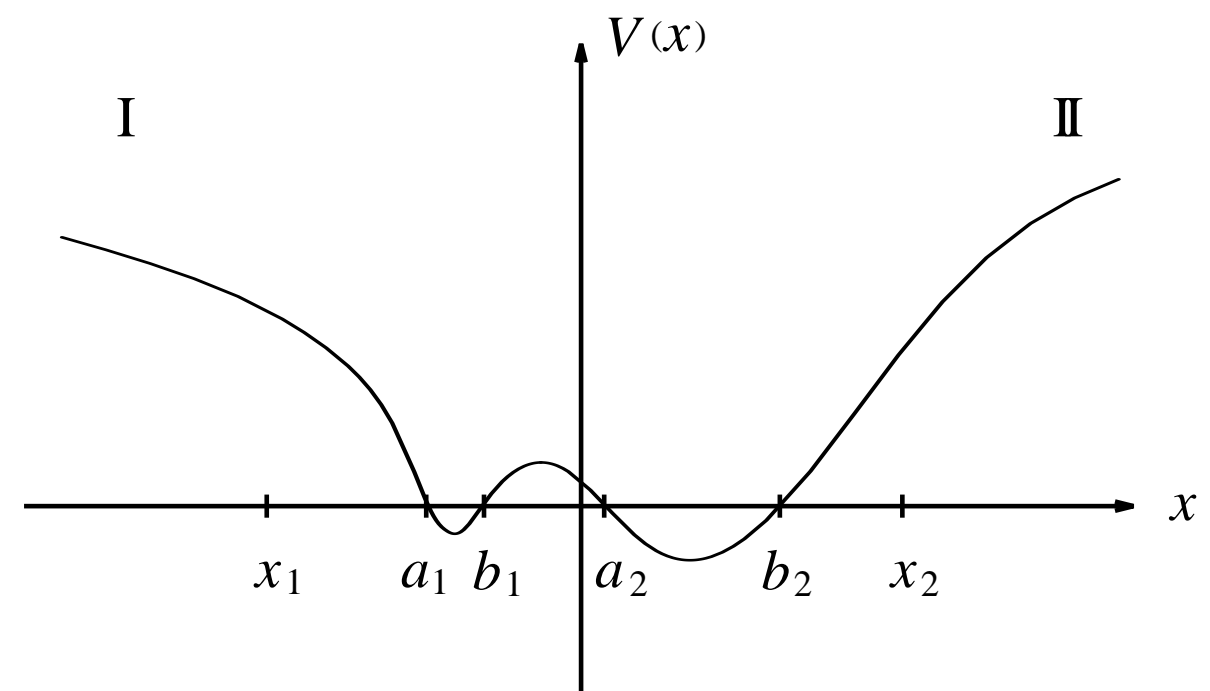

Fig.1 


\begin{tabular}{|c|c|c|c|}
\hline$\oint$ & $\begin{array}{ll}0 & 0 \\
0 & 0\end{array}$ & $\begin{array}{lll}0 & 0 & 0 \\
0 & 0 & 0\end{array}$ & $\begin{array}{llll}0 & 0 & 0 & 0 \\
0 & 0 & 0 & 0\end{array}$ \\
\hline$d$ & $\begin{array}{ll}0 & 0 \\
0 & \bullet\end{array}$ & $\begin{array}{lll}0 & 0 & 0 \\
0 & 0 & 0\end{array}$ & $\begin{array}{llll}0 & 0 & 0 & 0 \\
0 & 0 & 0 & 0\end{array}$ \\
\hline$\phi$ & $\begin{array}{ll}0 & 0 \\
0 & \bullet\end{array}$ & $\begin{array}{lll}0 & 0 & 0 \\
0 & \bullet & 0\end{array}$ & $\begin{array}{llll}0 & 0 & 0 & 0 \\
0 & \bullet & 0 & 0\end{array}$ \\
\hline$\phi$ & $\begin{array}{ll}0 & 0 \\
0 & \bullet\end{array}$ & $\begin{array}{lll}0 & 0 & 0 \\
0 & \bullet & \bullet\end{array}$ & $\begin{array}{llll}0 & 0 & \circ & 0 \\
0 & \bullet & \bullet & \bullet\end{array}$ \\
\hline
\end{tabular}

Fig.2 


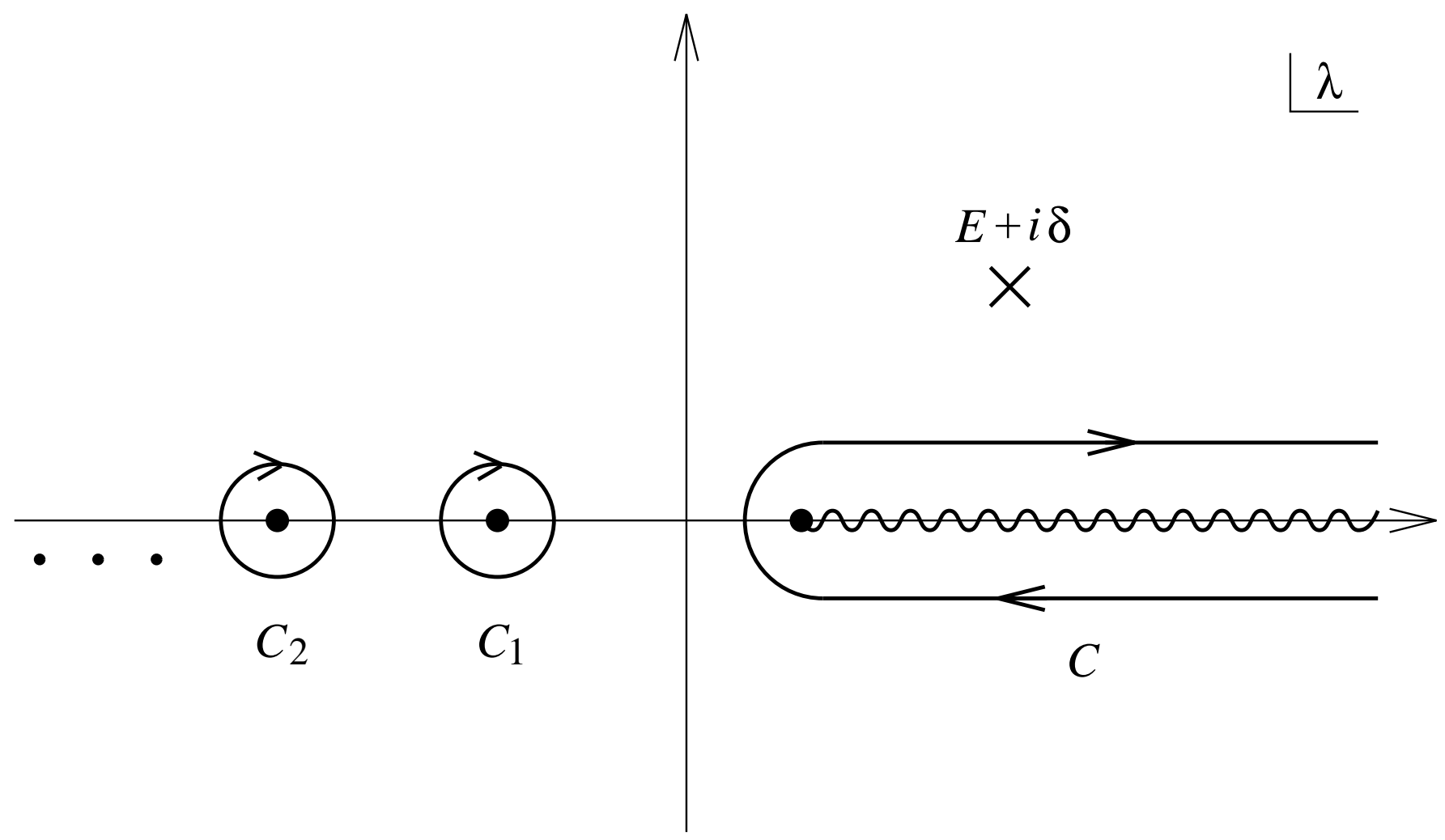

Fig.3 


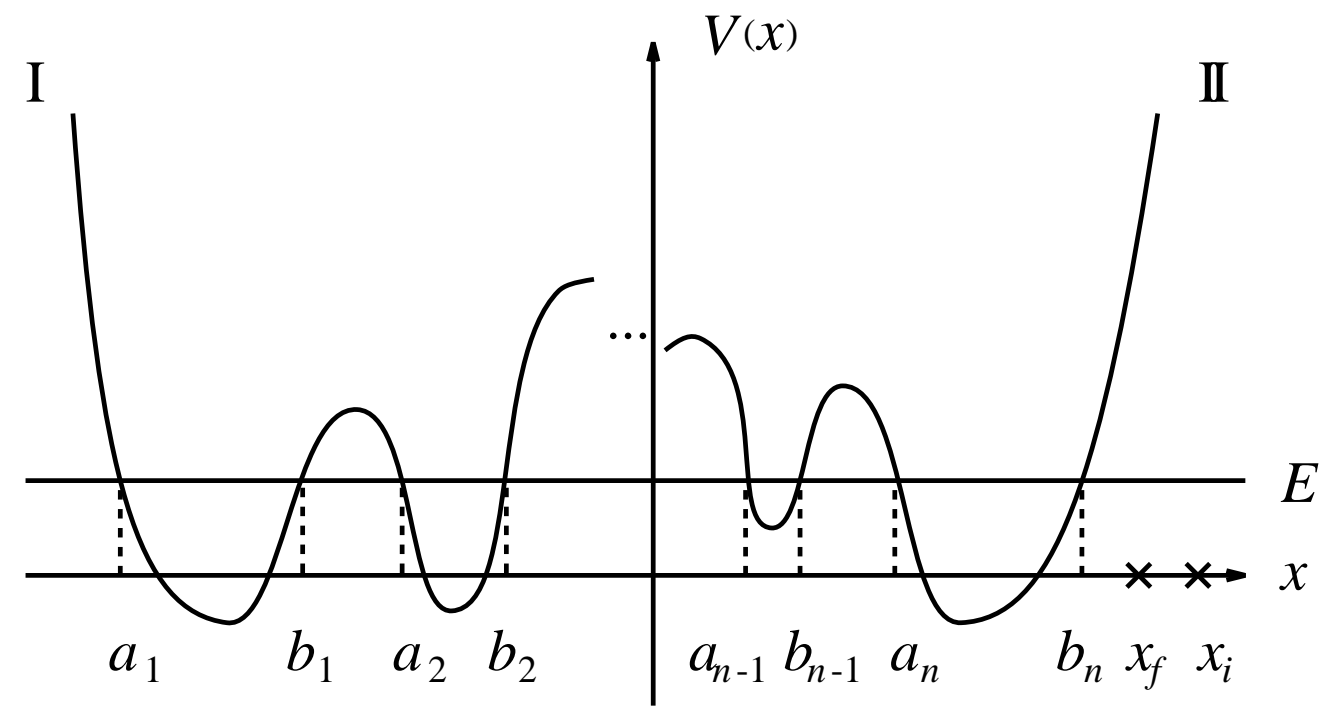

Fig.4 


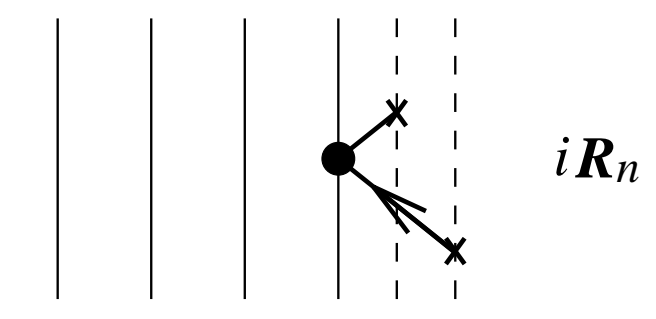

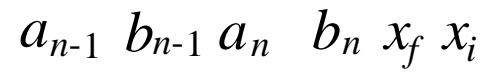

$\stackrel{\infty}{\infty}$

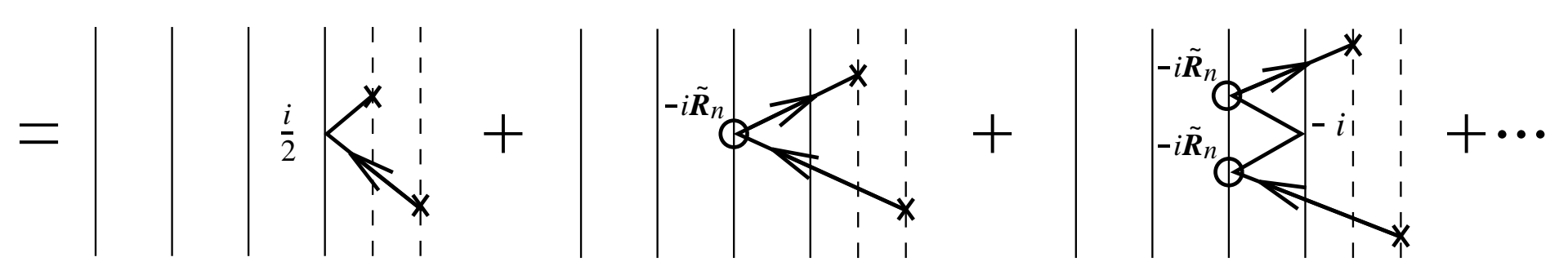

Fig.5 


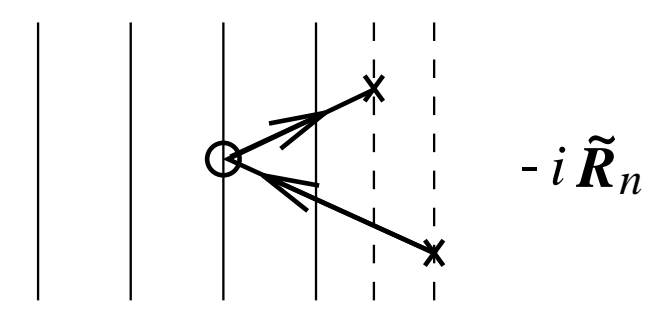

$\begin{array}{llllll}a_{n-1} & b_{n-1} & a_{n} & b_{n} & x_{f} & x_{i}\end{array}$

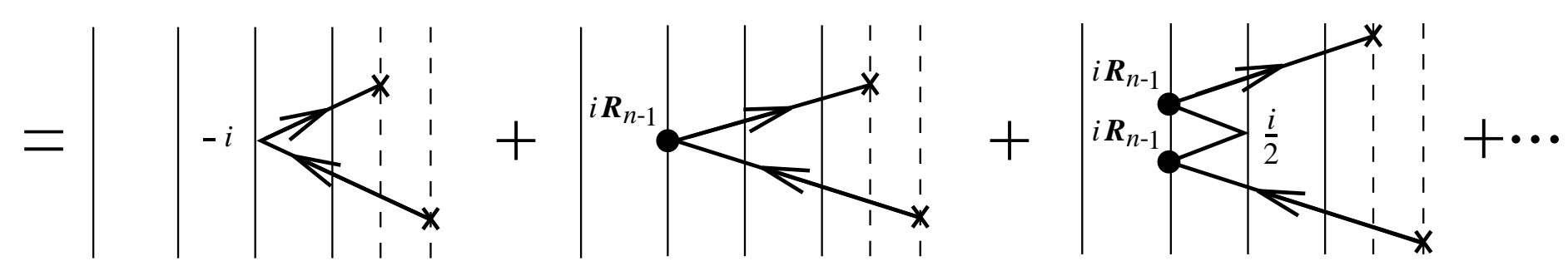

Fig.6 
(a)

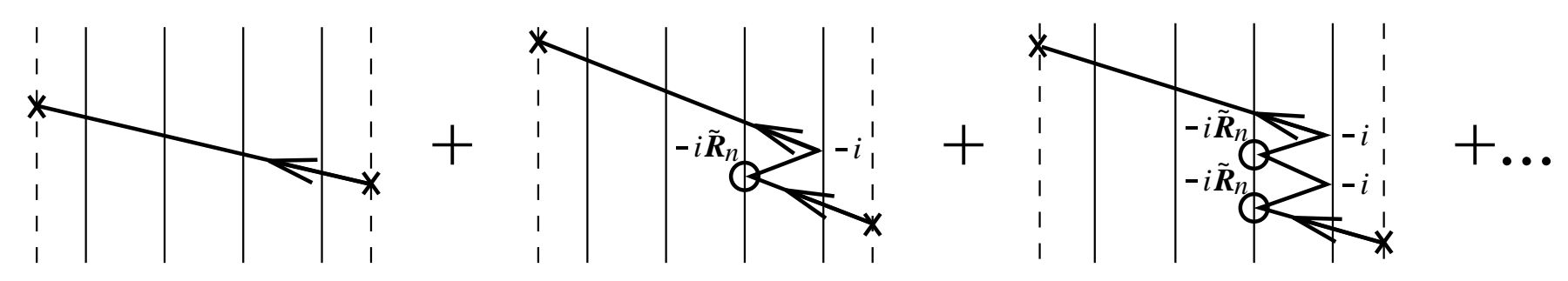

$\begin{array}{llllll}x_{f} & a_{n-1} & b_{n-1} & a_{n} & b_{n} & x_{i}\end{array}$

(b)
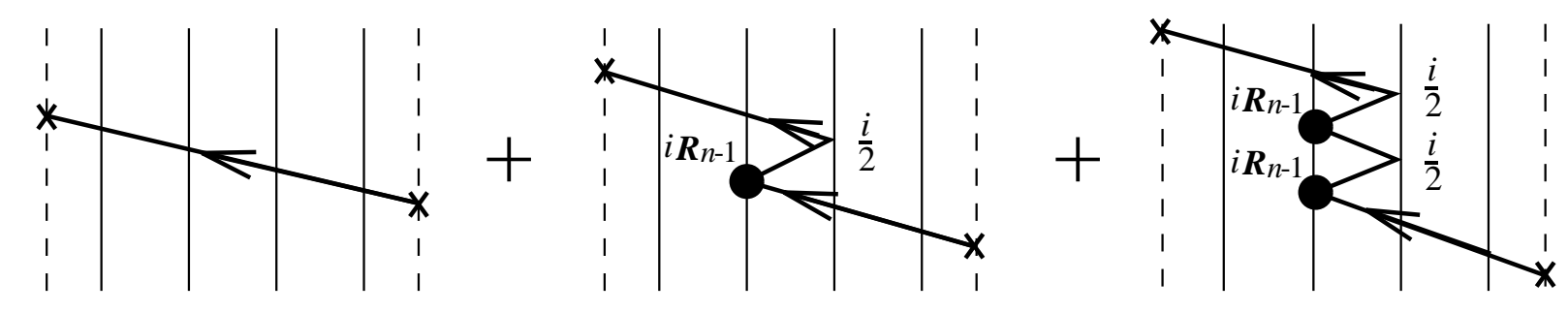

$+\ldots$

$\begin{array}{llllll}x_{f} & a_{n-1} & b_{n-1} & a_{n} & b_{n} & x_{i}\end{array}$

Fig.7 
(a)

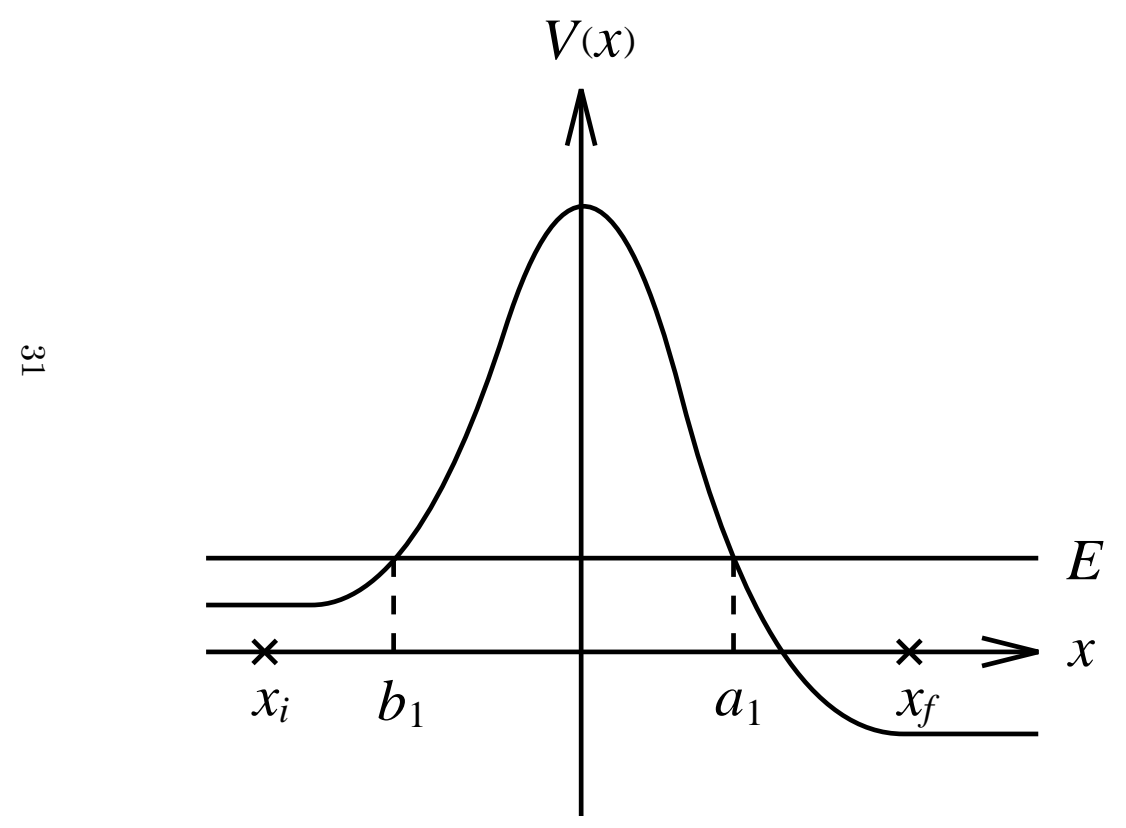

(b)

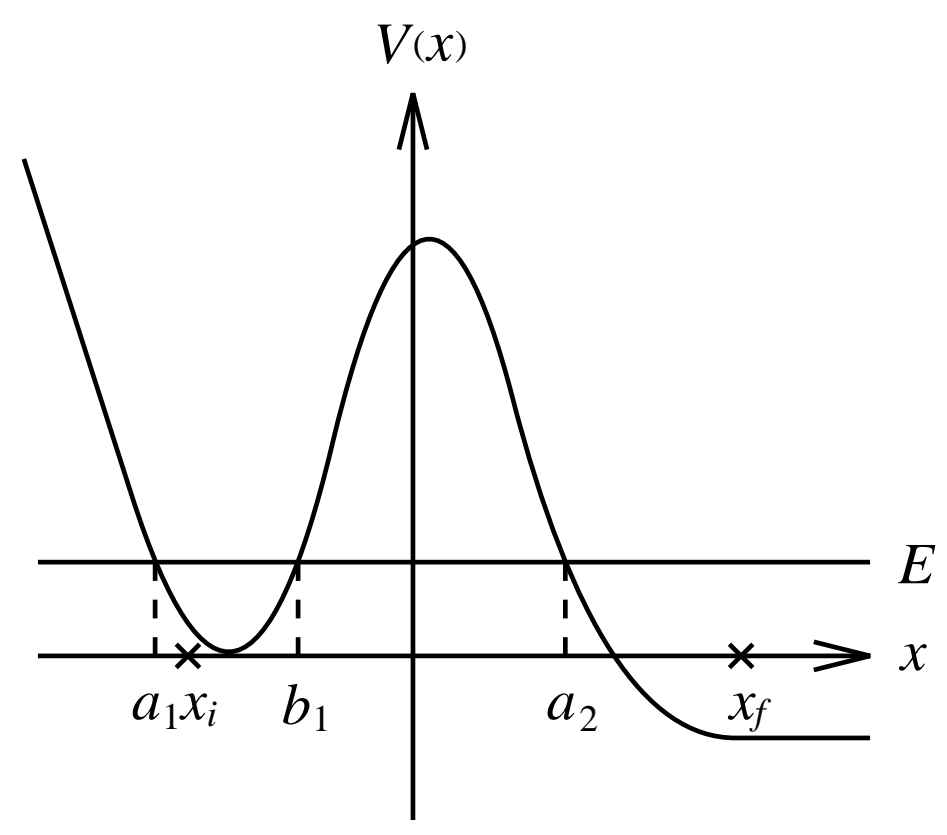

Fig.8 